Case report

\title{
Disseminated leishmaniasis caused by Leishmania tropica in HIV-positive patients in the Islamic Republic of Iran
}

\author{
S. Jafari, ${ }^{7}$ M. Hajiabdolbaghi, ${ }^{1}$ M. Mohebali, ${ }^{2}$ H. Hajjaran ${ }^{2}$ and H. Hashemian ${ }^{7}$
}

\section{Introduction}

Both cutaneous and visceral forms of leishmaniasis are prevalent in some parts of the Islamic Republic of Iran [1,2]. The Mediterranean type of visceral leishmaniasis (VL) is also reported sporadically throughout the country [3] with canines being the main animal reservoir hosts [4]. The cutaneous form of leishmaniasis is seen in 2 forms: anthroponotic and zoonotic. Anthroponotic cutaneous leishmaniasis (ACL) is caused by Leishmania tropica and is prevalent in many large- and medium-sized cities in the country $[1,5]$. Zoonotic cutaneous leishmaniasis (ZCL) is caused by L. major and is endemic in many foci in the north, east and south of the country [6]. Some studies have implicated L. tropica as another agent of $\mathrm{VL}$ in humans and dogs in reports from the north-west and south of the country $[4,7,8]$.

Few data are available, however, about hostimmunological response and parasite destruction when leishmaniasis is associated with immunosuppressants. At present, the majority of cases of HIVleishmaniasis co-infection reported in the Mediterranean basin were caused by L. infantum [9].

Disseminated leishmaniasis caused by L. tropica in patients with HIV infection is uncommon. Here, we report on 2 patients with HIV-leishmaniasis co-infection caused by L. tropica with generalized and multiple skin lesions and visceral involvement. This is the first such report from the Islamic Republic of Iran.

\section{Case reports}

\section{Case 1}

A 32-year-old man, an intravenous drug user with many tattoos, known for 5 months to be HIV-positive and with a history of hepatitis $\mathrm{C}$ and incompletely treated pulmonary tuberculosis, was admitted to our hospital in June 2003. The patient also had tuberculous abscess and many non-ulcerative leproid nodules distributed all over his face and he had deformed ears (Figure 1). His $\mathrm{CD}_{4}$ cell count was $180 / \mathrm{mm}^{3}$. Serological results for the detection of anti-Leishmania antibodies (DAT, IFA, rK39 dipstick) were negative $[2,10]$. Examination of peripheral blood smears disclosed no Leishman bodies. Blood culture in monophonic and biphasic media was negative, bone marrow aspiration and direct microscopic examination on Giemsa-stained smears revealed scant Leishmania amastigotes. Culture of bone marrow aspirate in monophonic and biphasic media was positive, and promastigotes were seen 10 days after inoculation.

Smears prepared from the skin lesions showed a great number of intracellularLeishman bodies within histiocytes, and even extracellular flagellated forms were observed (Figure 2). The culture was strongly positive 2 weeks after inoculation. To determine the species, following mass production in RPMI 1640 (Gibco), all of the promastigotes were analysed by a random amplification of polymorphic DNA polymerase chain reaction (RAPD-PCR) technique and the results compared with standard species of L. infantum (MCAN/IR/96/ LON49), L. tropica (MHOM/IR/99/ YAZ1) and L. major (MRHO/IR/75/ ER) using 4 RAPD-PCR primers, including AB1-07 (5' GGT GAC GCA G), A4 (5’ AAT CGG GCT G ), 327 (5' ATA CGG CGT C) and 329 (5' GCG AAC CTC C) in the School of Public Health, Tehran University of Medical Sciences [11,12]. Dr K.P. Chang from Chicago University confirmed the Leishmania identification with PCR-RFLP.

The patient was treated with antiretroviral drugs (zidovudine, lamivudine, nelfinavir) plus an antituberculosis regimen. We also started pentavalent antimony (Glucantime) $20 \mathrm{mg} / \mathrm{kg} /$ day for a period of 1 month and the patient was followed up during the treatment course.

The $\mathrm{CD}_{4}$ count of the patient reached $300 / \mathrm{mm}^{3} 2$ months after the treatment and a considerable improvement in skin lesions was seen although the lesions had not completely disappeared. 


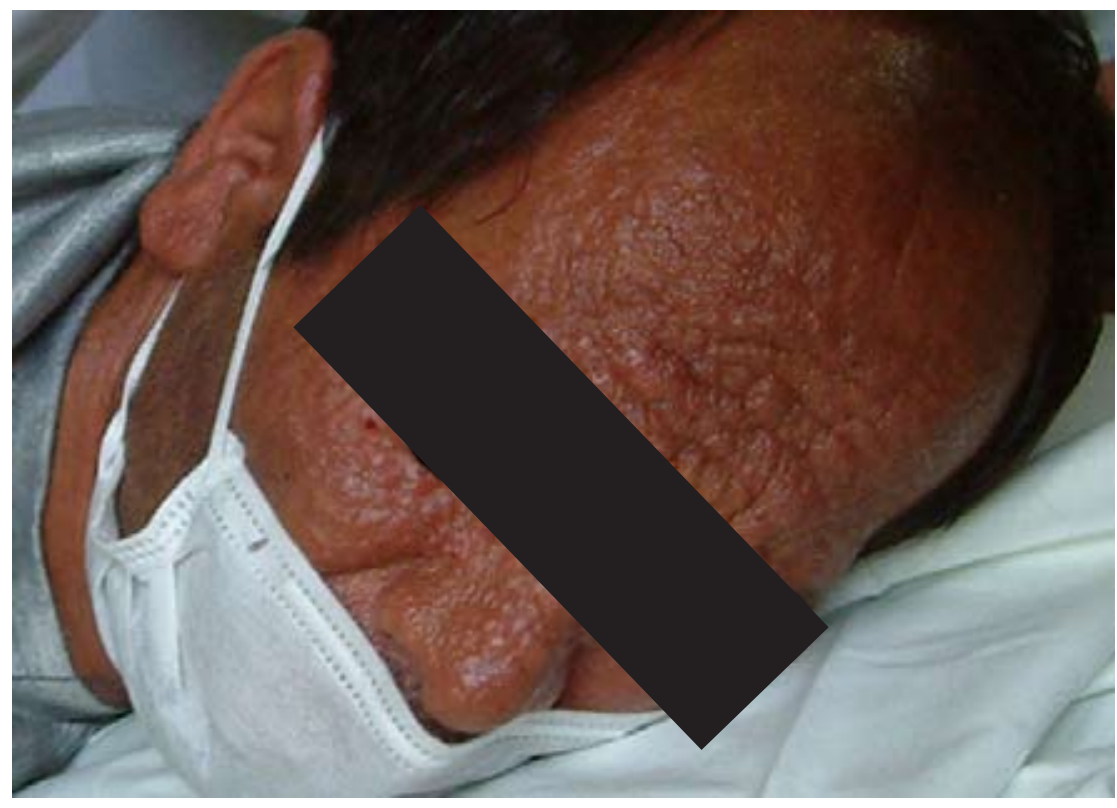

Figure 1 Distribution of the skin lesions on the face in case 1: erythematous papules and nodules with central ulceration covered with haematic crusts

\section{Case 2}

A 49-year-old man, who was an intravenous drug user and known to be HIVpositive, was admitted to our hospital in June 2006 with skin lesions distributed on his face and extremities.

Previously, he had a history of travel to Bam, a city in the south-east of the country which is endemic for ACL $[1]$.
He reported suffering multiple insect bites in Bam.

A few months after travelling, a small papular lesion appeared on his left ankle that gradually became larger. He had itching, erythematous, scaling plaque in same area, extending to the knees, elbow, flexor surface of the forearm, left side of the forehead, eyelid, nose and right ear.

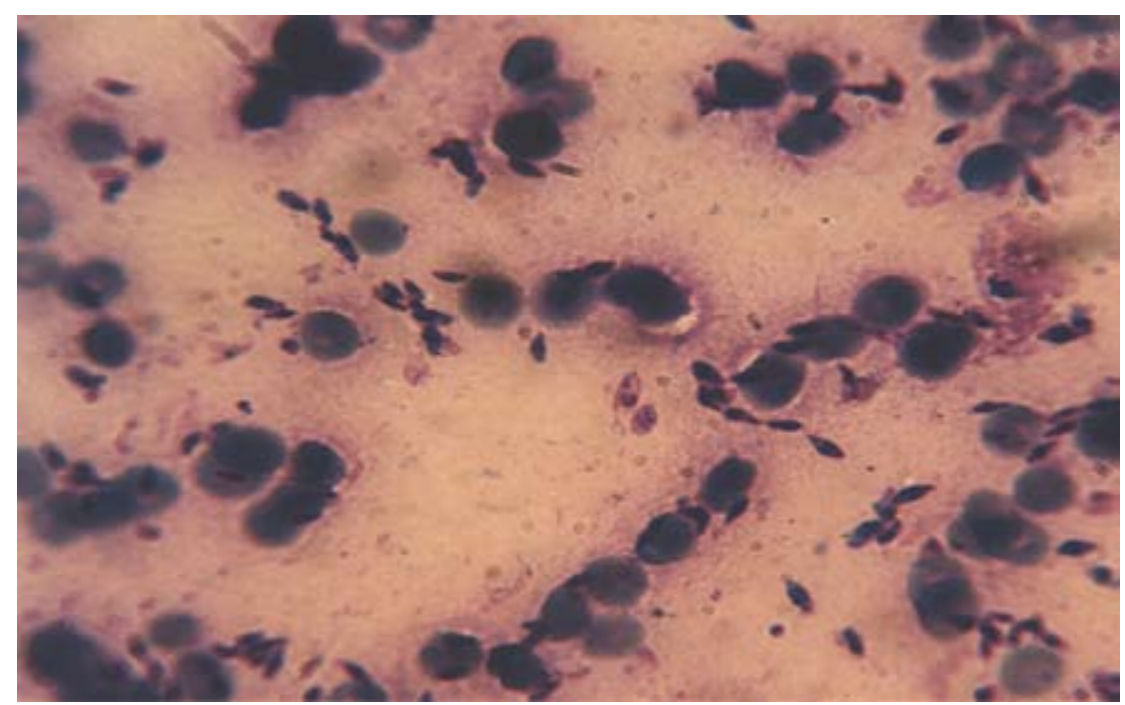

Figure 2 Leishman bodies in the Giemsa-stained smear, prepared from skin lesions of case $1(\times 1000)$
No fever, weight loss or other constitutional symptoms were observed. Except for multiple, small, mobile lymph nodes (maximum diameter $1.5 \mathrm{~cm}$ ) without tenderness detected in the cervical, axillary and inguinal chains, other physical findings were normal. Mild splenomegaly and a few small lymph nodes in the liver hilum and paraceliac area were reported using abdominal sonography. His $\mathrm{CD}_{4}$ cell count was $180 / \mathrm{mm}^{3}$.

Direct examination of the skin lesion biopsy showed Leishman bodies within histiocytes. Bone marrow aspiration and direct microscopic examination of Giemsa-stained smears revealed Leishmania sp. Culture of bone marrow aspiration in monophasic and biphasic media was positive and promastigotes were observed about 4 weeks after inoculation. Serological tests for the detection of anti-Leishmania antibodies (DAT, IFA, rK39 dipstick) were positive. To determine the species, we performed RAPD-PCR as described above $[11,12]$ and L. tropica was confirmed. Treatment with antiretroviral drugs (zidovudine, lamivudine, nevirapine) and pentavalent antimony (Glucantime) 20 $\mathrm{mg} / \mathrm{kg} /$ day was started.

The $\mathrm{CD}_{4}$ count of the patient rose to $210 / \mathrm{mm}^{3} 3$ months after treatment. A considerable improvement in skin lesions was also seen after treatment.

\section{Discussion}

Disseminated leishmaniasis is a granulomatous disease, clinically characterized by ulcerated skin and visceral involvement, whose clinical manifestations can progress spontaneously. Visceral involvement is often caused by $L$. donovani complex (L. donovani, L. infantum, and L. chagasi), endemic in the Middle East and Mediterranean regions [13]. VL is endemic in north-western and southern Islamic Republic of Iran, and mainly affects children; L. infantum is the dominant species $[1,3,4]$. ACL caused by $L$. tropica is endemic in some cities of our 
country $[1,5]$. In the Persian Gulf War (1990-91), L. tropica was the causative agent for leishmaniasis in American soldiers at bases in Bahrain and northern Saudi Arabia [14]. In recent years, Tehran and Bam have been recognized as endemic foci of ACL and interestingly, Patient 1 was resident in southern Tehran and Patient 2 had a history of travel to Bam.

There may be a very long incubation period of many years, so although the trip to Bam in Patient 2 may have been made a long time ago, he may have contracted leishmaniasis in that endemic area.

It is known that the pathogenesis of leishmaniasis depends on the interaction between antigen-presenting cells and the parasite involving immunological mechanisms [15]. Disseminated leishmaniasis has been described in many conditions with diminished immune response, including malignant disease, especially lymphoma, systemic lupus erythematous, renal transplantation anti-rejection therapy, during corticosteroid therapy, and, more recently, in patients with HIV infection [13]. Physicians should be aware of the atypical clinical manifestations of VL in HIVpositive patients, and should consider this diagnosis in cases of unexplained pyrexia, anaemia or pancytopenia, especially in endemic areas. Anaemia with or without leukopenia and thrombocytopenia may be found but may be by treatment such as zidovudine. Serological tests have low sensitivity and they are positive in $34 \%-55 \%$ of HIV-positive patients [16]. The clinical picture of disseminated cutaneous leishmaniasis in HIV-positive patients sometimes differs significantly from that of classic cutaneous leishmaniasis. In HIV-negative patients with classic cutaneous leishmaniasis, bone marrow, blood smear and culture are usually negative but in leishmaniasis-associated HIV, bone marrow, blood smear and culture may be positive, and skin lesions may be present in unusual locations. Typically, 2 or 3 relapses per patient occur in spite of the correct treatment [17].

The course of the disease is often related to the host immune response. The suppression of the immunological mechanisms of the host leads to facilitating the multiplication of the parasite and its dissemination [15,17]. Although the $\mathrm{CD}_{4}$ cell count decreased during an opportunistic infection in these $2 \mathrm{HIV}$ positive patients, the low immunity/ low $\mathrm{CD}_{4}$ count seems to be related to HIV infection as the primary factor for dissemination of leishmaniasis.

Cutaneous lesions in our HIVpositive patients were the first sign of visceral involvement. Skin lesions were quite variable: macules, papules, plaques nodules and ulcers with many amastigotes, particularly in the first patient, were observed. The presence of Leishmania amastigotes has previously been detected in healthy skin in patients with VL and HIV co-infection [17]. In our patients, treatment with pentavalent antimony led to the relative clearance of skin lesions but relapses in patients are common and a long follow-up period is needed.

The last, and perhaps most interesting, point about these cases is that they were caused by L. tropica. In previous reports, disseminated cutaneous forms of leishmaniasis were usually caused by $L$. donovani complex and they were accompanied by visceral involvement [18]. In a study carried out in southern Islamic Republic of Iran, 63 of the 64 children with VL had infection with $L$. infantum: there was, however, a single patient infected with L. tropica [7]. This patient was not immunocompromised and did not differ in any way from the other patients. Our cases are the first Iranian reports showing unusual cutaneous lesions with visceral involvement caused by L. tropica in HIV-leishmaniasis co-infection. This report shows that L. tropica can produce disseminated cutaneous leishmaniasis with visceral extension in HIV-positive patients.

We recommend blood samples collected from HIV-positive patients in the Islamic Republic of Iran are tested for Leishmania antigens to determine the prevalence of the infection, particularly in endemic foci of leishmaniasis.

\section{Acknowledgement}

Wethank DrK.P.Changforcarrying out PCR-RFLP using the primers designed from the nagt gene at the University of Chicago, United States of America.

\section{References}

1. Nadim A. Leishmaniasis. In: Azizi F, Janghorbani M, Hatam $\mathrm{H}$, eds. Epidemiology and control of common disorders in Iran, 2nd ed. Tehran, Endocrine \& Metabolism Research Center, Shaheed Beheshti University of Medical Sciences Press, 2000.

2. Mohebali M et al. Application of direct agglutination test (DAT) for the diagnosis and seroepidemiological studies of visceral leishmaniasis in Iran. Iranian journal of parasitology, 2006, 1(1):15-25.
3. Edrissian $\mathrm{GhH}$ et al. Visceral leishmaniasis: the Iranian experiences. Archives of Iranian medicine, 1998, 1(1):22-6.

4. Mohebali $\mathrm{M}$ et al. Epidemiological aspects of canine visceral leishmaniasis in the Islamic Republic of Iran. Veterinary parasitology, 2005, 129(3-4):243-51.

5. Yaghoobi-Ershadi MR et al. A new focus of cutaneous leishmaniasis caused by Leishmania tropica. Saudi medical journal, 2002, 23(3):291-4. 
6. Mohebali M et al. Characterization of Leishmania infection in rodents from endemic areas of the Islamic Republic of Iran. Eastern Mediterranean health journal, 2004, 10(4-5):591-9.

7. Alborzi AV et al. Leishmania tropica-isolated patient with visceral leishmaniasis in southern Iran. American journal of tropical medicine and hygiene, 2006, 74(2):306-7.

8. Hajjaran $\mathrm{H}$ et al. Leishmania tropica: another etiological agent of canine visceral leishmaniasis in Iran. Iranian journal of public health, 2007, 36(1):85-8.

9. Altés J et al. Visceral leishmaniasis: another HIV-associated opportunistic infection? Report of eight cases and review of the literature. AIDS, 1991, 5:201-7.

10. Edrissian $\mathrm{GhH}$ et al. Evaluation of rapid Dipstick rk39 test in diagnosis and serological survey of visceral leishmaniasis in humans and dogs in Iran. Archives of Iranian medicine, 2003, 6(1):29-31.

11. Noys HA et al. Appraisal of various random amplified polymorphic DNA-polymerase chain reaction primers for Leishmania identification. American journal of tropical medicine \& hygiene, 1996, 55:98-105
12. Hajjaran $\mathrm{H}$ et al. Identification of Leishmania species isolated from human cutaneous leishmaniasis, using random amplified polymorphic DNA (RAPD-PCR). Iranian journal of public health, 2004, 33(4):8-15.

13. Gilles HM, ed. Protozoal diseases. London, Arnold, 1999:452.

14. Magill AJ et al. Visceral infection caused by Leishmania tropica in veterans of Operation Desert Storm. New England journal of medicine, 1993, 329(20):1503-4.

15. Kharazmi A et al. T-cell response in human Leishmaniasis. Immunology letter, 1999, 65:105-8.

16. El Safi SH et al. A comparison of the direct agglutination test and enzyme- linked immunosorbent assay in the sero-diagnosis of leishmaniasis in the Sudan. Transactions of the Royal Society of Tropical Medicine and Hygiene, 1989, 83:334-7.

17. Alvar J. Leishmaniasis and AIDS co-infection: the Spanish example. Parasitology today, 1994, 10:160-3.

18. Herrera E et al. The presence and significance of Leishmania in mucocutaneous biopsies from HIV+ patients with visceral leishmaniasis. European journal of dermatology, 1996, 6:51.

\section{Joint EMRO/TDR Small Grants Scheme for Operational Research in Tropical and Other Communicable Diseases: 18th call for proposals 2010}

The Eastern Mediterranean Regional Office (EMRO) of the World Health Organization (WHO) in collaboration with the UNICEF/UNDP/World Bank/WHO Special Programme for Research and Training in Tropical Diseases (TDR) is pleased to announce the 18th call for proposals of the Small Grants Scheme for Operational Research in Tropical and other Communicable Diseases for the year 2010. The scheme is co-funded by the WHO/EMRO and the UNICEF/ UNDP/World Bank/WHO Special Programme for Research and Training in Tropical Diseases (TDR). The deadline for application is 31 March 2010. The Research Proposal Form and further information about the Small Grants Scheme is available at: http://www.emro.who.int/TDR/pdf/TDR_Small_Grants_Scheme_010.pdf 